\title{
ASSESSMENT OF MAXILLARY IMPACTED CANINES USING PANORAMIC RADIOGRAPH AND CONE-BEAM COMPUTED TOMOGRAPHY
}

\author{
Shaimaa Elmarhoumy* and Nahla Elsayed Gomaa**
}

\begin{abstract}
Aim: Assess the labio-palatal position of maxillary impacted canines (MICs) on CBCT and their mesiodistal position on panoramic radiographs.

Materials and Methods: This retrospective radiographic study reviewed 70 radiographs with MICs (40 bilaterally and 30 unilateral). Radiographs with, odontoma, mesiodenses, or cleft lip and palate were excluded. The mesiodistal position of the MICs was classified into four sectors on panoramic radiograph while their labiopalatal position were evaluated on CBCT. The correlation between panoramic sectors location and $\mathrm{CBCT}$ results was examined using the chi-square test.

Results: Labial positioned MICs on CBCT were more frequent in panoramic sector 1, while palatally positioned MICs were more frequent in sectors 4 . The association between sectors of the MICs on panoramic images and the labio-palatal position of the MICs on CBCT was statistically significant $(P<0.001)$.
\end{abstract}

Conclusions: This study showed that, sector location on panoramic radiographs might be used for prediction of labiopalatal position of MICs.

KEY WORDS: Impaction, maxillary canine, panorama, CBCT.

\section{INTRODUCTION}

Maxillary permanent canine is considered as the second most commonly impacted tooth (the first most commonly impaction is the third molar). Impacted canine may be located palatal or buccal to dental arch or in line with the arch. Buccal and in line with arch impaction can be attributed to early loss of deciduous teeth that may lead to arch length loss, crowding tendency, or space deficiency of dental arch. While, palatal impaction may be due to genetic cause or loss of lateral incisor guidance on its distal surface ${ }^{[1]}$. Panoramic radiograph has been used in orthodontics as a routine method to diagnose and identify canine impaction by locating cusp tip of canine in relation to root of lateral incisor, state of tooth eruption, tooth angulation,

\footnotetext{
* Lecturer of Orthodontics, Faculty of dentistry, Tanta University, Egypt.

** Assistant Professor of Orthodontics, Faculty of Dentistry, Tanta University, Egypt.
} 
third molar prognosis, plan treatment and also to asses treatment results ${ }^{[2]}$. Limitation of panoramic radiograph can be referred to blurring of image error of distortion projection overlapping of adjacent structures to impacted canine leading to difficulty in assessing impacted canine labiopalatally and incisors root resorption ${ }^{[3,4]}$. Limitations of $\mathrm{CT}$ to be used to analyze impacted canine include higher exposure dose related to panoramic radiograph and it is relatively expensive ${ }^{[5]}$. These limitations can be overcome by using cone beam computed tomography (CBCT) as it is considered the most accurate method to locate impacted canine, mesial angulation of root, root resorption and root contact.

Several studies about reliability of panoramic radiograph and $\mathrm{CBCT}$ on specific conditions - such as canine and third molar impaction, supernumerary teeth, and root resorption - were done. These studies revealed that $\mathrm{CBCT}$ gives more reliable information than panoramic radiograph in these conditions ${ }^{[6,7]}$

The aim of our study is to assess the use of panoramic radiograph and $\mathrm{CBCT}$ to localize maxillary impacted canine.

\section{MATERIALS AND METHODS}

The subjects in this study were selected with impacted maxillary canine with age above 12 years (46 female and 24 male) involved 40 bilaterally impacted canine and 30 unilateral impacted canines (18 at right side and 12 at left side). The sample size and power analysis were calculated using EpiInfo software statistical package created by World Health organization and center for Disease Control and Prevention, Atlanta, Georgia, USA version 2002. The criteria used for sample size calculation were as follows: $95 \%$ confidence limit, $80 \%$ power of the study, The sample size was found at $\mathrm{N}=70$ participants. The Exclusion criteria were systemic diseases, odontoma, mesiodenses, or cleft lip and palate. For each patient, panoramic radiograph was made using a Proline $\mathrm{XC}^{*}$ radiography unit and CBCT scan was acquired with a DCT Pro** Scanning parameters was $90 \mathrm{kVp}, 24 \mathrm{~s}, 4 \mathrm{~mA}$, voxel size $0.4 \mathrm{~mm}$ and field of view $20 \times 19 \mathrm{~cm}$. [8]. A CBCT scanner was used to identify the exact locations of the impacted maxillary canines. Using CBCT data, each sample was classified according to the labiopalatal location of the maxillary impacted canine into labial and palatal groups.

The mesiodistal positions of the impacted maxillary canines were classified into a panoramic sector using Ericson and Kurol's method [9] (fig.1). Each measurement was recorded by single examiner. To evaluate intraexaminer reliability, data were reinvestigated after an interval of 1 month. The correlation between the sector location on the panoramic radiographs and the labiopalatal position on the CBCT images was investigated using the chisquare test.

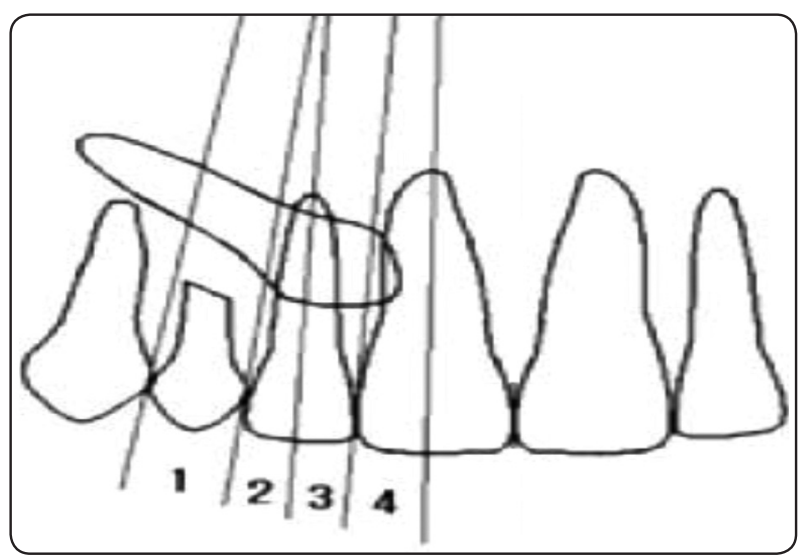

Fig. (1) Sector Locations of maxillary canine cusp tips on panoramic radiographs. Sector 1: area distal to the line tangent to the distal heights of contour of the lateral incisor crown and root. Sector 2: area bounded by sector 1 and the long axis of the lateral incisor. Sector 3: area bounded by sector 2 and a line tangent to the mesial heights of contour of the lateral incisor crown and root. Sector 4: area bounded by sector 3 and the long axis of the central incisor.

* (Planmeca, Helsinki, Finland)

** (Vatech Co., Hwasung, Republic of Korea) 


\section{RESULTS}

Twenty four of 37 labially maxillary impacted canines were in sector 1 , while 13 MICs were in sector 2. As regard to the palatal group, 22 were in sector 4 while 11 MICs were in sector 3. A statistically significant correlation was observed between the sectors and the labiopalatal positions of the canines $(\mathrm{P}<0.001$; Table I). Labially impacted maxillary canines were more frequently observed in sector 1 (fig 2), while palatally impacted maxillary canines were more frequently observed in sector 4 (fig 3).
TABLE (I) Relationship between sector location on panoramic radiographs and labiopalatal position on CBCT images.

\begin{tabular}{|c|c|c|c|c|c|c|c|}
\hline \multirow{2}{*}{$\begin{array}{l}\text { Impaction } \\
\text { position }\end{array}$} & \multicolumn{4}{|c|}{ Sectors } & \multirow{2}{*}{ Total } & \multirow{2}{*}{$\begin{array}{l}\text { Chi- } \\
\text { square }\end{array}$} & \multirow{2}{*}{$P$ value } \\
\hline & 1 & 2 & 3 & 4 & & & \\
\hline Labial & 24 & 13 & 0 & 0 & 37 & \multirow{3}{*}{29.001} & \multirow{3}{*}{$0.001 *$} \\
\hline Palatal & 0 & 0 & 11 & 22 & 33 & & \\
\hline Total & 24 & 13 & 11 & 22 & 70 & & \\
\hline
\end{tabular}
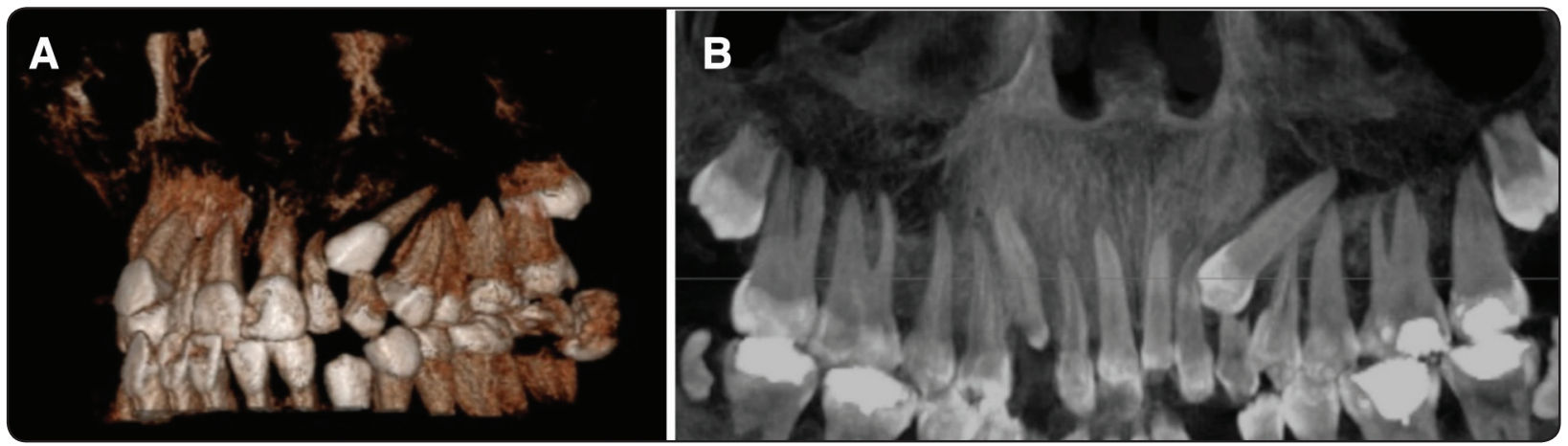

Fig. (2) (A) CBCT image showing labially impacted maxillary left canine. (B) panoramic radiographic image showing labially impacted maxillary left canine in sector 1 .
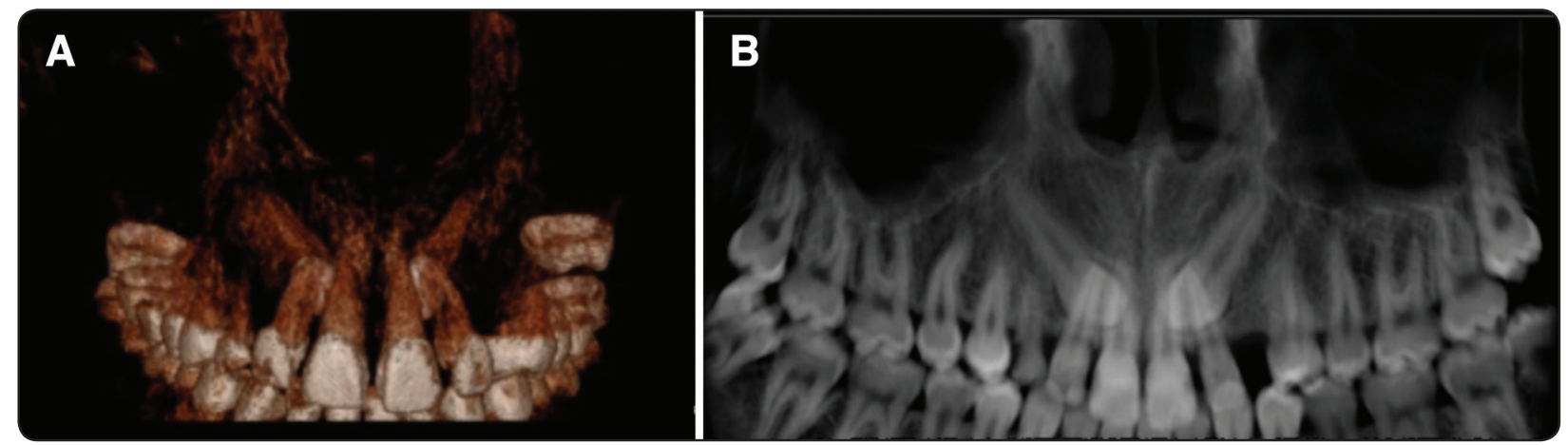

Fig. (3) (A) CBCT image showing palatally impacted maxillary canines (B) panoramic radiographic image showing palatally impacted maxillary canines in sector 4 . 


\section{DISCUSSION}

Buccal impaction of permanent maxillary canines can be attributed to crowding of dental arch due to arch length deficiency either due to genetic or local cause ${ }^{[1]}$. Palatal impaction of permanent maxillary is due to lack of guidance to canine as in peg shaped, short root or missing lateral incisor ${ }^{[1,4]}$.

CBCT was used to overcome limitations panoramic and CT radiographs.

The aim of this study was to correlate between panoramic radiograph and $\mathrm{CBCT}$ in localization of maxillary impacted canine.

Panoramic and $\mathrm{CBCT}$ radiographs were used to locate mesiodistal positions of the impacted maxillary canines. Impacted canines were classified into a panoramic sector using Ericson and Kurol's method ${ }^{[9]}$. Results showed that statistically significant correlation between sectors and labiopalatal positions of the canines was found.

These results are in line with those done by Serrant et al in that CBCT is more accurate than 2D panoramic radiograph in localization of maxillary impacted canines ${ }^{[10]}$.

Regard sector location, Lindauer et al and Warford et al stated that sector location has a great effect on prediction of impacted canine more than angulation has. They found that labial impaction of canine using CBCT were frequent on panoramic radiograph in sector 1,2 and 3, while, palatally impacted canines were more frequent in sector 4 using panoramic radiograph. These results coincide with those found in this study ${ }^{[3,11]}$.

This study suggests that sector location using panoramic radiograph can help in determining labiopalatal position of maxillary impacted canines, to minimize using CBCT to decrease the cost for patient.

\section{CONCLUSION}

Based on the findings of the current study, the following conclusions can be suggested:

- Labially impacted maxillary canines as seen on CBCT were more frequent observed in sector 1 , while palatally impacted maxillary canines were more frequent seen in sectors 4 , on panoramic radiographs.

- Sector location on panoramic radiography can be used to predict the labiopalatal position of maxillary impacted canines.

\section{REFERENCES}

1. Orton HS, Garvey MT and Pearson MH. Extrusion of ectopic maxillary canine using a lower removable appliance. Am J Orthod Dentofacial Orthop 1995; 107: 349-59.

2. Wyatt DL, Farman AG, Orbell GM, Silveira AM and Scarfe WC. Accuracy of dimensional and angular measurements from panoramic and lateral oblique radiographs. Dentomaxillofac Radiol.1995;24:225-31.

3. Lindauer SJ, Rubenstein LK, Hang MW, Andersen WC and Isaacson RJ. Canine impaction identified early with panoramic radiographs. J Am Dent Assoc 1992; 123:91-2.

4. Alqerban A, Jacobs R, Fieuws S and Willem G. Comparison of two cone beam computed tomographic systems versus panoramic imaging for localization of impacted maxillary canines and detection of root resorption. Eur J Orthod 2011; 33:93-102.

5. Pittayapat P, Willems G, Alqerban A,Coucke W, Rotta F R, Souza P C,Westphalen F H and Jacobs R. Agreement between cone beam computed tomography images and panoramic radiographs for initial orthodontic evaluation.Oral Surg Oral Med Oral Pathol Oral Radiol 2014; 117:111-9.

6. Scarfe WC, Farman AG and Sukovic P. Clinical applications of cone beam computed tomography in dental practice. JCDA 2006; 72:75-80.

7. Alqerban A, Jacobs R, keirsbilck P, Aly M, Swinnen S, Fieuws S and Willems G. The effect of using CBCT in the diagnosis of canine impaction and its impact on the orthodontic treatment outcome. J Orthod Sci. 2014; 3: 34-40. 
8. Jung YH, Liang H, Benson BW, Flint DJ and Cho BH. The assessment of impacted maxillary canine position with panoramic radiography and cone beam. Dentomaxillofac Radiol J.2012;41,356-60.

9. Ericson S and Kurol J. Early treatment of palatally erupting maxillary canines by extraction of the primary canines. Eur J Orthod. 1988; 10:283-95
10. Serrant PS, Grant T, Mclntyre and Thomson DJ. Localization of ectopic maxillary canines - is CBCT more accurate than conventional horizontal or vertical parallax?.JO 2014; 41:13-8.

11. Warford JH, Grandhi RK and Tira DE. Prediction of maxillary canine impaction using sectors and angular measurement. J Am Dent Assoc 2003; 124:651-5. 